\title{
Application of the Lognormal Raindrop Distribution to Differential Reflectivity Radar Measurement $\left(Z_{D R}\right)$
}

\author{
GRAHAM FEINGOLD AND ZEV LEVIN \\ Department of Geophysics and Planetary Sciences, Raymond and Beverley Sackler Faculty \\ of Exact Sciences, Tel Aviv University, Ramat Aviv, Israel 69978
}

(Manuscript received 18 July 1986, in final form 21 November 1986)

\begin{abstract}
Use of the lognormal form of raindrop size distributions in simulations of differential reflectivity $\left(Z_{D R}\right)$ measurements is investigated. Using two remotely measured variables and an empirical relation, the three parameters of the lognormal distribution can be deduced and the spectrum integrated to obtain rain rate. This is demonstrated by a simulation of the $Z_{D R}$ method using ground-based drop size distributions. Drop axis ratio and sampling time effects are also investigated and results compared to those obtained using a gamma distribution. It is shown that the lognormal representation is easily adaptable for use in the $Z_{D R}$ method. Using our dataset, we show that the lognormal size distribution provides lower average absolute deviations of theoretically determined rain rates from actual ones $(10.7 \%)$ than those obtained using either the exponential (41.0\%) or gamma distributions $(11.8 \%)$.
\end{abstract}

\section{Introduction}

In a previous paper, Feingold and Levin, (1986 hereafter FL), showed that the lognormal distribution function provides a good fit to raindrop size distributions (DSDs) in Israel and is particularly useful for tracing the evolution of DSDs. In addition, FL, following Ulbrich's (1981) analysis of the suitability of various functional forms in remote measurement techniques, presented preliminary results strongly suggesting that the lognormal presentation would be advantageous in the dual-parameter radar measurement of rainfall. While it is recognized that the effects of variations in the shape of DSDs are by no means the only source of error in measured rainfall parameters, their influence can at least be significantly reduced when a more accurate representation of the DSD is used (see Ulbrich, 1981; Ulbrich and Atlas, 1984). It is with this aim in mind that we investigate here the applicability of the lognormal distribution in differential reflectivity radar measurements of rainfall $\left(Z_{D R}\right)$. In the $Z_{D R}$ method, two orthogonally polarized reflectivity components, $Z_{H}$ and $Z_{V}$, are measured and used to determine two parameters of the size spectrum. Once the latter is known, it can be integrated to obtain the corresponding rain rate or any other rainfall parameter. For details of the $Z_{D R}$ technique, the reader is referred to Seliga and Bringi (1976). Since only two parameters are measured by the $Z_{D R}$ radar, the DSD can at best be described by a two-parameter function. Seliga and Bringi (1976) suggested the exponential distribution, whereas Ulbrich (1983a), recognizing the need for a more flexible function, capable of representing curved spectra, proposed use of the modified gamma distribution:

$$
N(D)=N_{0} D^{\mu} e^{-(3.67+\mu) D / D_{0}} \quad\left[\mathrm{~m}^{-3} \mathrm{~mm}^{-1}\right]
$$

which reduces to the exponential distribution when $\mu$ $=0$. Although the gamma distribution has three parameters, $N_{0}, \mu$ and $D_{0}$ (the median volume diameter), it has been used in its two-parameter form with $\mu \mathrm{em}$ pirically related to $N_{0}$ by an expression of the form:

$$
N_{0}=A e^{b_{\mu}} \quad\left[\mathrm{m}^{-3} \mathrm{~mm}^{-1}\right]
$$

(Ulbrich, 1983a). Based on their measurements in Israel, FL showed that the lognormal distribution can be reduced to its two-parameter form, thus making it a viable alternative to the exponential and gamma distributions. The lognormal distribution is given by

$$
N(D)=\frac{N_{T}}{\sqrt{2 \pi} \ln \sigma D} e^{-\ln ^{2}\left(D / D_{g}\right) / 2 \ln ^{2} \sigma} \quad\left[\mathrm{m}^{-3} \mathrm{~mm}^{-1}\right]
$$

with three parameters, $N_{T}$, the drop concentration $\left(\mathrm{m}^{-3}\right), D_{g}$, the geometric mean diameter $(\mathrm{mm})$ and $\sigma$, the breadth parameter. FL showed that although $\sigma$ can vary somewhat for any particular rain rate, on average, $\sigma$ is independent of rain rate and has a value of approximately 1.4. Similar evidence has been given by Markowitz (1976), who found that $\sigma$ is equal to about 1.39 over a wide range of rain rates $\left(2.5-150 \mathrm{~mm} \mathrm{~h}^{-1}\right)$.

In a simulation of the $Z_{D R}$ method, reflectivity parameters $Z_{H}, Z_{V}$ and $Z_{D R}\left[=10 \log \left(Z_{H} / Z_{V}\right)\right]$ have been calculated from distrometer data sampled in central Israel (4601 1-min samples) and the behavior of these parameters analyzed in terms of their dependences on the lognormal parameters. Using a simple procedure, analogous to that proposed by Seliga and Bringi (1976), it will be shown that the two-parameter lognormal dis- 
tribution can reduce the inherent errors in rain rate estimation introduced by assumption of the exponential form. Moreover, the lognormal distribution produces results on a par with, or even better than, those reported by workers using the gamma distribution.

\section{Theory}

Falling drops attain the approximate shape of oblate spheroids due to the balance between hydrostatic, surface tension and dynamic stresses. Drop shapes are therefore parameterized according to their minor to major axis ratios $(a / b)$. Using Rayleigh scattering theory for the case of oblate spheroids, as developed by Gans (1912), it can be shown (see Seliga and Bringi, 1976) that the horizontal and vertical reflectivity components, $Z_{H}$ and $Z_{V}$ are given by

$Z_{H}=\frac{\Lambda^{4}}{|K|^{2} \pi^{5}} \int_{0}^{D_{\max }} \sigma_{H}(a / b) N\left(D_{e q}\right) d D_{e q} \quad\left[\mathrm{~mm}^{6} \mathrm{~m}^{-3}\right]$

$Z_{V}=\frac{\Lambda^{4}}{|K|^{2} \pi^{5}} \int_{0}^{D_{\max }} \sigma_{V}(a / b) N\left(D_{e q}\right) d D_{e q} \quad\left[\mathrm{~mm}^{6} \mathrm{~m}^{-3}\right]$

where $D_{e q}$ is the equivalent diameter of an oblate spheroid, $D_{\max }$ the maximum drop diameter and $\sigma_{H, V}$, (not to be confused with the breadth parameter, $\sigma$ ), the scattering cross sections for an oblate spheroid at horizontal and vertical polarizations. Here $\Lambda$ is the radar wavelength and $K$, a function of the refractive index of water. The differential reflectivity $\left(Z_{D R}\right)$ is defined by

$$
Z_{D R}=10 \log \left(Z_{H} / Z_{V}\right)
$$

With $N(D)$ given by the exponential distribution (Eq. 1 with $\mu=0$ ) we find that $Z_{D R}$ is independent of $N_{0}$ and can therefore be used to determine the median volume diameter, $D_{0}$. Either of the $Z_{H}$ or $Z_{V}$ measurements can then be used to determine the second parameter of the distribution, $N_{0}$. With $N_{0}$ and $D_{0}$ known, the spectrum can be integrated to obtain $R$ :

$$
R=\frac{\pi}{6} \int_{0}^{D_{\max }} N\left(D_{e q}\right) D_{e q}{ }^{3} v\left(D_{e q}\right) d D_{e q} .
$$

With the inadequacy of the exponential drop-size distribution now well established, the expression for $N\left(D_{e q}\right)$ in Eq. (6) should be replaced by either the gamma or the lognormal distributions. We propose substitution of the lognormal distribution (3) with $\sigma$ $=$ constant into Eqs. (4a) and (4b) as follows:

$\frac{Z_{H}}{N_{T}}=\frac{\Lambda^{4}}{|K|^{2} \pi^{5}} \int_{0}^{D_{\max }} \frac{\sigma_{H}(a / b)}{\sqrt{2 \pi} \ln \sigma D_{e q}} e^{-\ln ^{2}\left(D / D_{g}\right) / 2 \ln ^{2} \sigma} d D_{e q}$

$\left[\mathrm{mm}^{6}\right]$

$$
\frac{Z_{V}}{N_{T}}=\frac{\Lambda^{4}}{|K|^{2} \pi^{5}} \int_{0}^{D_{\max }} \frac{\sigma_{V}(a / b)}{\sqrt{2 \pi} \ln \sigma D_{e q}} e^{-\ln ^{2}\left(D / D_{g}\right) / 2 \ln ^{2} \sigma} d D_{e q}
$$

Note that $N_{T}$ can be removed from the integrals so that $Z_{D R}$ is a function of $D_{g}$ only. It can easily be shown (see e.g., FL) that the median volume diameter $\left(D_{0}\right)$ is related to $D_{g}$ by the following relation:

$$
D_{0}=D_{g} e^{31 \ln ^{2} \sigma} \text {. }
$$

Measurement of $Z_{D R}$ thus enables direct determination of $D_{g}$ or $D_{0} . N_{T}$ can then be calculated from the measurements of either $Z_{H}$ [Eq. (7a)] or $Z_{V}$ [Eq. (7b)]. The method is completely analogous to that proposed by Seliga and Bringi (1976), but has the advantage that it avails itself of the superior fit of the lognormal distribution (albeit a two parameter version). Theoretical curves showing the dependence of $Z_{D R}$ and $10 \log \left(Z_{H} /\right.$ $N_{T}$ ) on $D_{g}$ are shown in Fig. $1 \mathrm{a}, \mathrm{b}$ for various values of $\sigma$. For the reader more familiar with $D_{0}$ as the size parameter, these curves are reproduced in Fig. $2 \mathrm{a}, \mathrm{b}$ with $Z_{D R}$ and $Z_{H} / N_{T}$ this time plotted vs $D_{0}$. Superimposed on the curves in Fig. 1a, b are the data points representing two parameter lognormal fits to the measured spectra. The method of fit is one which calculates $D_{g}$ and $N_{T}$ from the third and sixth moments of the spectrum (liquid water content and radar reflectivity, respectively), while keeping $\sigma$ at a fixed value (in this case, 1.4). The curves are obtained by Gaussian-quadrature numerical integration of Eqs. (7a) and (7b) for $D_{\max }=7 \mathrm{~mm}$, a wavelength of $10 \mathrm{~cm}$, a refractive index of water of $m=8.99+1.47 i$ and axis ratios of Pruppacher and Pitter (1971). The latter are similar to the equilibrium values calculated by Green (1975). The work of Pruppacher and Beard (1970) and that of Cooper et al. (1983) suggest that drops are more oblate. On the other hand, Jameson and Beard (1982) argue that collision induced oscillations encountered in natural rainfall increase the axis ratios (decrease the oblateness) to values above those at equilibrium. In light of the disagreement in the published literature, we have chosen the Pruppacher and Pitter data which lie within the range of proposed values. For an analysis of the sensitivity of the $Z_{D R}$ method to drop oscillations (periodic variations in the axis ratio), see Seliga et al. (1984). Figs. 1a and $1 \mathrm{~b}$ show how when the values of $Z_{D R}$ and $Z_{H} / N_{T}$ are plotted against $D_{g}$ for all the data points (each point representing $1 \mathrm{~min}$ of rain), the points follow the theoretical curves for $\sigma=1.4$ throughout the range. In fact, in the case of Fig. 1b, there is no discernible deviation of the data points from the theoretical curve.

\section{Results and discussion}

In order to check the validity of this approach, the DSD data sampled in Hadera, Israel will be used. (For details, see FL). With dual polarization measurements 

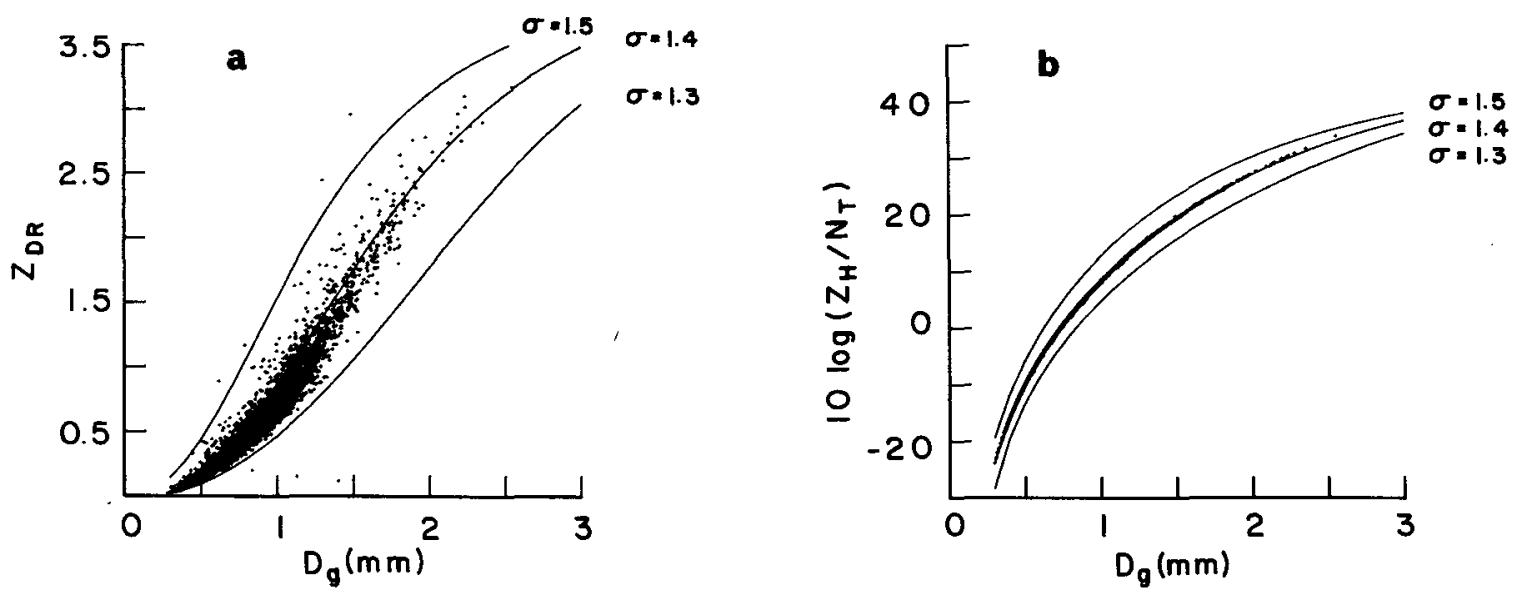

FIG. 1a. Theoretical curves of (a) $Z_{D R}$ and (b) $10 \log \left(Z_{H} / N_{T}\right)$ vs $D_{g}$ for various values of $\sigma$. Superimposed on the curves are data points representing third and sixth moment weighted fits to the observed spectra. Note that the points in (b) are coincident with the theoretical curve.

not at our disposal, the only way of assessing the viability of this method is to change the inverse problem to the forward problem-i.e., $Z_{D R}$ and $Z_{H}$ will be assumed to be known (their values will be calculated directly from the measured spectra) and the theoretical curves in Figs. 1a and $1 \mathrm{~b}$ will be used to determine distribution parameters $D_{g}$ and $N_{T}$ for a constant value of $\sigma$. This approach has the salutary effect of focusing attention on the effects of distribution shape on the measurement of rainfall.

Using the values of $Z_{D R}$ and $Z_{H}$ computed directly from the spectra, the rain rates will now be calculated. These theoretically estimated rain rates $\left(R_{T}\right)$ will then be compared to the actual rain rates $\left(R_{D}\right)$ as calculated from the distrometer measurements. $R_{T}$ is plotted vs $R_{D}$ in Fig. 3. The least-squares fit to the points is represented by the line:

$$
R_{T}=1.099 R_{D}+0.108
$$

with a standard error of estimate (SEE) of $0.581 \mathrm{~mm}$ $\mathrm{h}^{-1}$. The absolute average deviation of $R_{T}$ from $R_{D}$, defined by $\Delta=\left|R_{T}-R_{D}\right| / R_{D}$, is $22.3 \%$.

The dependence of $\Delta$ on the values of $D_{g}$ and $N_{T}$ can be considered as follows. From Figs. $1 \mathrm{a}$ and $1 \mathrm{~b}$ it is clear that if $D_{g}$ is erroneously overestimated by $+\Delta D_{g}$ then Eq. (7b) will overestimate the value of $Z_{H} / N_{T}$, or underestimate $N_{T}$ by a value of $-\Delta N_{T}$. In other words, an error of $+\Delta D_{g}$ incurs an error of $-\Delta N_{T}$, and because $R_{T}$ is directly dependent on both $D_{g}$ and $N_{T}$ the errors tend to counter one another. This can be analyzed using the theoretical form of the lognormal distribution in Eq. (6). Dropping the subscript "eq" for brevity, we can write:

$R=\frac{\pi}{6} \int_{0}^{D_{\max }} N(D) D^{3} v(D) d D=c_{n} N_{T} D_{g}^{3.67}$

$\left[\mathrm{mm} \mathrm{h}^{-1}\right]$
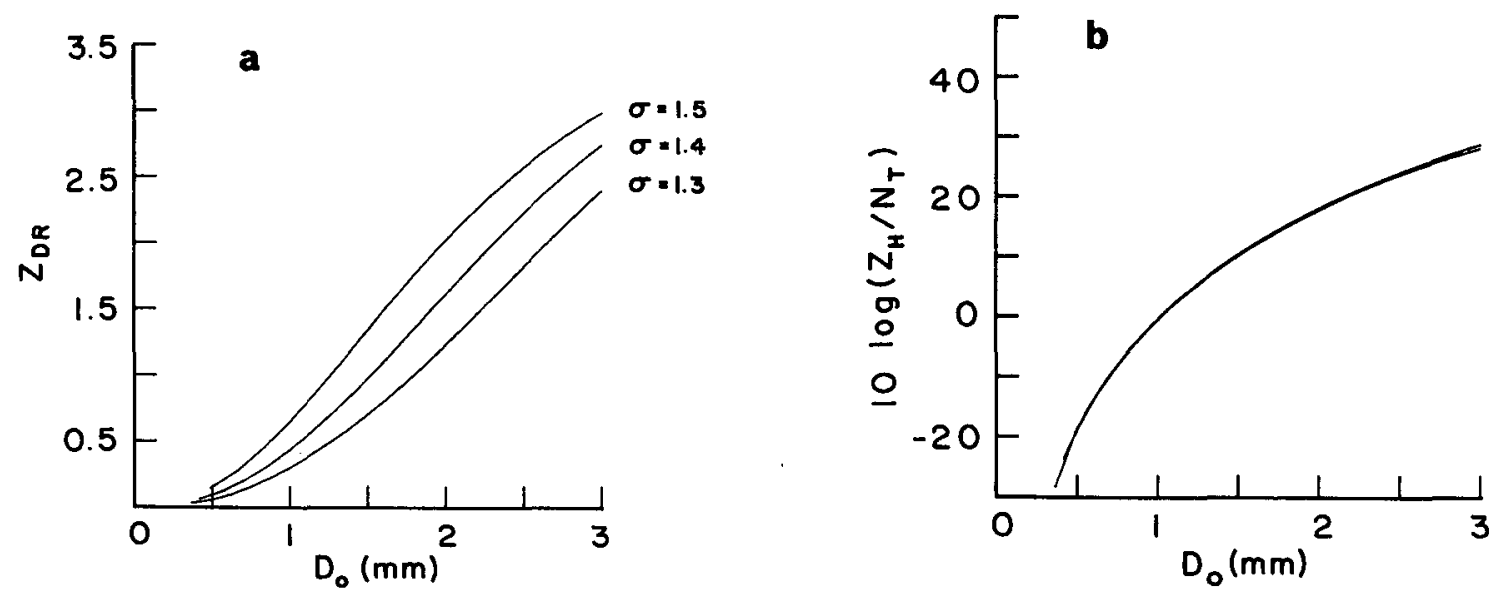

FIG. 2. Theoretical curves of (a) $Z_{D R}$ and (b) $10 \log \left(Z_{H} / N_{T}\right.$ ) vs $D_{0}$ for the various values of $\sigma$. In the case of $(b)$ the curves almost coincide. 


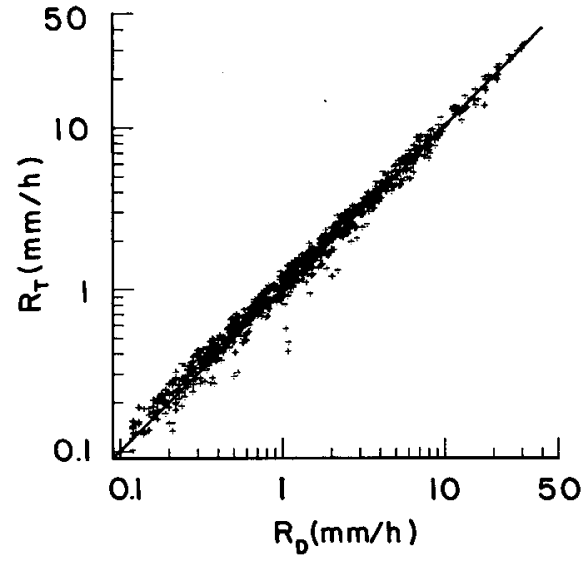

FIG. 3. $R_{T}$ (the theoretically determined rain rates) plotted as a function of $R_{D}$ (actual rain rates). Each point represents a 5-min averaged distribution.

where $c_{n}=7.1 \cdot 10^{-3} e^{6.73 \ln ^{2} \sigma}$ and $v(D)$ in Eq. (10) has been approximated by the power law $v(D)=3.77 D^{0.67}$ ( $v$ in $\mathrm{m} \mathrm{s}^{-1}$ and $D$ in $\mathrm{mm}$ ) after Atlas and Ulbrich (1977). Following the previous discussion,

$$
R_{T}=c_{n}\left(N_{T}-\Delta N_{T}\right)\left(D_{g}+\Delta D_{g}\right)^{3.67}
$$

can then be written as:

$$
\Delta=\frac{R_{T}}{R_{D}}-1=\left(1-\Delta N_{T} / N_{T}\right)\left(1+\Delta \dot{D}_{g} / D_{g}\right)^{3.67}-1 .
$$

Clearly, $\Delta$ is inversely proportional to both $D_{g}$ and $N_{T}$, with a much stronger dependence on $D_{g}$. Consequently, $\Delta$ is expected to decrease with increasing $R$ (see also Ulbrich and Atlas, 1984). Since the vast majority of our 4601 data points ( 1 min sampling time) are for rain rates below $5 \mathrm{~mm} \mathrm{~h}^{-1}$, and only a few points have rain rates greater than $40 \mathrm{~mm} \mathrm{~h}^{-1}$ (see Fig. $1, F L)$, a value of $\Delta=22 \%$ is a promising result.

\section{a. Effect of increasing sampling time}

In using distrometer measured DSDs in this analysis, we implicitly assume that they are representative of the drop spectra in the volume of air scanned by the radar. However, the sampling volumes of the radar and distrometer differ significantly $\left(\mathrm{km}^{3} \mathrm{vs} \mathrm{m}^{3}\right.$, for a sampling time of $1 \mathrm{~min}$ ). In order to equate these volumes, various workers (see, for example, Austin, 1981) have proposed equating large-volume averaged radar samples with long-time averaged distrometer samples. The results so far presented have been for 1 min time averaged samples. Goddrad and Cherry (1984) have used 30 -sec averaged samples, whereas Ulbrich (1983b) used 7-min averages in obtaining his results (quoted later in section 3 ). The results obtained when a sampling time of $5 \mathrm{~min}$ is used on our data are as follows; the equation for the best fit line to $R_{T}$ vs $R_{D}$ is given by

$$
R_{T}=1.033 R_{D}+0.057
$$

with an SEE of 0.469 . The corresponding value of $\Delta$ is reduced to $13.9 \%$. Note that these results are a great deal better than those previously obtained. Clearly, the choice of sampling time has a significant bearing on the results. In fact, if the sampling time is further increased to $7 \mathrm{~min}$ then the value of $\Delta$ is reduced to $12.1 \%$. At low rain rates, where the number of sampled drops is often small, longer sampling times are required in order to obtain better defined distributions. Thus, use of 5-min averaged distributions in these simulations is justified considering that the majority of rain rates in this dataset are less than $1 \mathrm{~mm} \mathrm{~h}^{-1}$.

\section{b. Comparison with a two parameter gamma distri- bution}

Widespread use has been made of the gamma distribution of $Z_{D R}$ measurements, and this discussion would not be complete without a comparison of the results obtained for a gamma distribution with those quoted above for the lognormal representation. We use a gamma distribution [Eq. (1)] with $\mu=$ constant rather than the $N_{0}-\mu$ relationship [Eq. (2)] since it has been shown by $\mathrm{FL}$ as well as Chandrasekhar and Bringi (1986) that such a relation may lead to inaccuracies, especially for large $\mu$. Fitting the gamma distribution to our data using the weighted moment method suggested by Ulbrich (1983a) and then plotting the derived values of $\mu$ as a function of $R_{D}$, it was found that a least-squares fit to the data points yielded a value of $\mu$ $=6.5$. Using the latter and a sampling time of $5 \mathrm{~min}$, we find that

$$
R_{T}=0.868 R_{D}+0.118
$$

with a SEE of 1.157 and $\Delta=12.3 \%$. Table 1 summarizes the values of $\Delta$ and the parameters of the leastsquares regression lines $(A$, the slope parameter and $B$, the intercept parameter) for both the lognormal and gamma distributions for sampling times of 1 and 5

TABLE 1. A comparison of the values of $\Delta$ (average absolute deviation), slope and intercept parameters of the regression lines $(A$ and $B$, respectively) and standard error of estimate (SEE) for the lognormal and gamma distributions using sampling times of $1 \mathrm{~min}$ and $5 \mathrm{~min}$ and the axis ratios of PP and PB.

\begin{tabular}{cccccc}
\hline \hline $\begin{array}{c}\text { Sampling } \\
\text { time (min) }\end{array}$ & $A$ & $B$ & $\Delta \%$ & SEE & $\begin{array}{c}\text { Axis } \\
\text { ratio }\end{array}$ \\
\hline \multicolumn{5}{c}{ Lognormal $(\sigma=1.4)$} \\
1 & 1.086 & 0.003 & 15.5 & 0.716 & PB \\
5 & 1.027 & 0.005 & 10.2 & 0.383 & PB \\
1 & 1.099 & 0.108 & 22.3 & 0.581 & PP \\
5 & 1.033 & 0.057 & 13.9 & 0.469 & PP \\
& & Gamma $(\mu=6.5)$ & & \\
1 & 0.934 & 0.127 & 19.1 & 1.310 & PB \\
5 & 0.869 & 0.106 & 11.1 & 1.150 & PB \\
1 & 0.934 & 0.163 & 22.1 & 1.280 & PP \\
5 & 0.868 & 0.118 & 12.3 & 1.157 & PP \\
\hline
\end{tabular}


min. Also included is a comparison of the results produced by the Pruppacher and Beard (1970) (henceforth PB) axis ratios with those obtained using Pruppacher and Pitters' (1971) (henceforth PP) data. The following points are evident from Table 1.

(i) Although the value of $\Delta$ obtained using the gamma distribution is a little lower than that of the lognormal (12.3\% as opposed to $13.9 \%$ for a sampling time of $5 \mathrm{~min}$ ), the parameter $A$ of the former deviates from the ideal by $-13 \%$ (i.e., underestimates $R_{D}$ ) whereas that of the latter deviates by only $+3 \%$ (overestimates $R_{D}$ ). In the case of the more oblate PB axis ratios, however, the value of $\Delta$ obtained using the lognormal is lower than that for the gamma distribution ( $10.3 \%$ as opposed to $11.1 \%$ ) while the $A$ parameters are similar to those found for the PP axis ratios.

(ii) For both distributions lower values of $\Delta$ are obtained when 5-min averaged distributions are used rather than 1-min averages.

(iii) Regardless of which axis ratios are employed, the lognormal values of SEE tend to be lower than those of the gamma distribution.

(iv) The more oblate $a / b$ ratios of PB produce better results than those obtained using the $P P$ data. This can be ascribed to the fact that the success of the $Z_{D R}$ method rests entirely on the degree of oblateness of the drops. Beard (1984) has suggested that axis ratios are in fact a function of rain rate. This premise is based on the assumption that higher rain rates are characterized by more frequent drop collisions and more substantial deviations from equilibrium axis ratios. Adopting this premise, the drop distributions with low rain rates which constitute the majority of this dataset should therefore exhibit axis ratios close to their equilibrium values, thus enhancing the success of the $Z_{D R}$ method.

(v) The parameter $A$ of the least-squares fit can be "corrected" by adjusting the values of $\mu$ to 4.5 and $\sigma$ to 1.37 (see Table 2). In this case (using the PP axis ratios), we find that $\Delta$ (lognormal) $=10.7 \%$ and $\Delta$ (gamma) $=11.8 \%$ (i.e., the lognormal presentation performs better. Table 2 also includes a comparison with the values obtained using an exponential distri-

TABLE 2. A comparison of the optimum values of $\Delta$ (average absolute deviation), slope and intercept parameters of the regression lines ( $A$ and $B$, respectively) and standard error of estimate (SEE) for the lognormal, gamma and exponential distributions, using a $5 \mathrm{~min}$ sampling time and the axis ratios of $\mathrm{PP}$.

\begin{tabular}{lcccc}
\hline \hline & $A$ & $B$ & $\Delta \%$ & SEE \\
\hline $\begin{array}{c}\text { Lognormal } \\
\sigma=1.37\end{array}$ & 0.990 & 0.028 & 10.7 & 0.425 \\
$\begin{array}{c}\text { Gamma } \\
\mu=4.3\end{array}$ & 0.996 & 0.002 & 11.9 & 0.418 \\
Exponential & 1.289 & 0.117 & 41.0 & 0.621 \\
\hline
\end{tabular}

bution). Clearly, the latter produces unacceptably high values of $\Delta(41.0 \%)$.

(vi) The results presented in Table 2 seem to suggest that for application to $Z_{D R}$, the weighted moment fit to DSDs proposed by Ulbrich (1983a) places too much emphasis on the large drop component of the spectrum and overestimates $\mu$ (i.e., produces distributions which are too narrow). Decreasing this value of 4.5 (broadening the spectrum) corrects the gamma distribution's consistent underestimation of rain rate evident in Table 1. Similarly, Table 2 also suggests that the method of weighted fit proposed by FL, which places homogeneous weighting throughout the spectrum, tends to overestimate the rain rate deduced by these $Z_{D R}$ simulations by overestimating $\sigma$ (i.e., producing distributions which are too broad). The above discussion underlines the importance of choosing a correct value of the breadth parameter (be it $\sigma$ or $\mu$ ) if satisfactory results are to be obtained.

Note that two factors have been isolated in the above discussion, one being the effect of drop distribution shape and the other, the effect of the drop axis ratios on $Z_{D R}$ measurements. If we use $\Delta$ as a measure of the success of the method, then DSD effects show (section 3a) that $\Delta$ is indirectly dependent on rain rate and hence that better results would be achieved under high rain rate conditions. The effect of the axis ratios works in exactly the opposite direction. If one adopts Beard's (1984) assumption that the greater the rain rate, the more frequent the collisions and the less oblate the drops, then one would expect the effectiveness of the $Z_{D R}$ method to be reduced under such conditions. On the other hand, recent measurements by Caylor and Illingworth (1986) suggest that large $Z_{D R}$ signals (up to $6 \mathrm{dBZ}$ ) may be obtained at high rain rates. To explain this phenomenon, they conclude that large drops are more oblate than their equilibrium values. Clearly, more measurements of the shapes of natural rain drops at various rain rates are required to resolve this issue.

We can compare the aforementioned results to those found by other workers. Ulbrich (1983) has used a three-parameter form of the gamma distribution and the empirical relation relating $N_{0}$ to $\mu$ [Eq. (2)] and found a value of $\Delta=11.9 \%$. Using a two-parameter gamma distribution with a constant value of $\mu=2$, Ulbrich (1983b) found a deviation of $13.5 \%$. Goddard and Cherry (1984) used a gamma distribution with a value of $\mu=5$ and applied it to their ground-based distrometer measurements of spectra. They found that the systematic offset of $R_{T}$ from $R_{D}$ is $1 \%$, with a standard deviation of $14 \%$. Using a gamma distribution and an $N_{0}-\mu$ relationship, Goddard and Cherry found an offset of $6 \%$ and a deviation of the order of $14 \%$. It should be noted that both these datasets appear to have proportionately higher numbers of points at high rain rates than did our dataset. Thus, the values of $\Delta$ obtained using the lognormal distribution function com- 
pare very favorably (especially for 5-min averaged distributions) with these aforementioned results.

\section{Conclusions}

The evidence produced here of the applicability of the lognormal distribution to dual parameter remote measurement techniques corroborates our preliminary results as discussed in FL. We show here how a twoparameter lognormal function can be adapted for use in the dual parameter $Z_{D R}$ method. With a constant value of $\sigma$ in Eq. $(7 \mathrm{a}, \mathrm{b}), Z_{D R}$ measurements can be used to calculate $D_{g}$. Then $N_{T}$ can in turn be calculated from $Z_{H}$. Rain rates determined using Eq. (6) and the deduced values of $D_{g}$ and $N_{T}$ are shown to deviate from distrometer measured rain rates by between $13.9 \%$ and $22.3 \%$, for $5-\mathrm{min}$ and $1-\mathrm{min}$ averaged distributions, respectively, with a standard error of estimate of about $0.5 \mathrm{~mm} \mathrm{~h}^{-1}$. These values compare favorably with those obtained using the gamma size distribution representation. If, on the other hand, the value of $\sigma$ is adjusted to 1.37 (i.e., a little lower than the empirically determined value), $\Delta$ is reduced to only $10.7 \%$ (using the $P P$ axis ratios and a sampling time of $5 \mathrm{~min}$ ). This result was not equaled using a gamma distribution with $\mu=$ constant.

The results of these simulated $Z_{D R}$ methods in which measured DSDs are used to deduce $Z_{H}, Z_{V}$ and $Z_{D R}$ values rather than using the real radar measured reflectivity parameters are sensitive to: (i) the relative number of events at high rain rates, (ii) the distribution sampling time, (iii) the drop axis ratios $(a / b)$ and (iv) the choice of the distribution breadth parameter.

The lognormal distribution has a wide range of applications in rainfall studies and, as shown here, can also be successfully employed in the $Z_{D R}$ and other dual-parameter techniques. Although our results show that both the gamma and lognormal distributions perform admirably well, and are certainly superior to the exponential form, we feel, on the basis of these results and those previously presented by Feingold and Levin (1986), that the lognormal distribution is to be preferred.

\section{REFERENCES}

Atlas, D., and C. W. Ulbrich, 1977: Path and area-integrated rainfall measurement by microwave attenuation in the $1-3 \mathrm{~cm}$ band. J. Appl. Meteor., 16, 1322-1331.
Austin, P. M., 1981: On deducing rainfall from radar reflectivity measurements. Conf. Radar Meteorology, Boston, Amer. Meteor. Soc., 200-207.

Beard, K. V., 1984: Oscillation models for predicting raindrop axis and backscatter ratios. Radio Sci., 19, 67-74.

Caylor, I. J., and A. J. Illingworth, 1986: Observations of the growth and evolution of raindrops using dual-polarization radar. Conf. Radar Meteorology, Snowmass, Amer. Meteor. Soc., 88-91.

Chandrasekhar, V., and V. N. Bringi, 1986: Estimators of dropsize distribution parameters from raindrop sampling devices. Conf. Radar Meteorology and Cloud Physics, Snowmass, Amer. Meteor. Soc., JP154-JP157.

Cooper, W. A., V. N. Bringi, V. Chandrasekhar and T. A. Seliga, 1983: Analyses of raindrop parameters using a 2-D precipitation probe with application of differential reflectivity. Conf. Radar Meteorology, Edmonton, Amer. Meteor. Soc., 488-493.

Feingold, G., and Z. Levin, 1986: The lognormal fit to raindrop spectra from frontal convective clouds in Israel. J. Climate Appl. Meteor., 25, 1346-1364.

Gans, R., 1912: Über die form ultramikroskopischer goldteilchen. Ann. Phys. (Leipzig), 37, 881-900.

Goddard, J., and S. Cherry, 1984: Quantitative precipitation measurements with dual linear polarisation data. Conf. Radar $\mathrm{Me}$ teorology, Zurich, Amer. Meteor. Soc., 352-357.

Green, A. W., 1975: An approximation for the shapes of large raindrops. J. Appl. Meteor., 14, 1578-1583.

Jameson, A. R., and K. V. Beard, 1982: Raindrop axial ratios. $J$. Appl. Meteor., 21, 257-259.

Markowitz, A., 1976: Raindrop size distribution expressions. J. Appl. Meteor., 15, 1029-1031.

Pruppacher, H. R., and K. V. Beard, 1970: A wind tunnel investigation of the internal circulation and shape of water drops falling at terminal velocity in air. Quart. J. Roy. Meteor. Soc., 96, 247256.

- and R. L. Pitter, 1971: A semi-empirical determination of the shape of cloud and raindrops. J. Atmos. Sci., 28, 86-94.

Seliga, T. A., and V. N. Bringi, 1976: Potential use of radar-differential reflectivity measurements at orthogonal polarizations for measuring precipitation. J. Appl. Meteor., 15, 69-76.

,- K. Aydin and V. N. Bringi, 1984: Differential reflectivity and circular depolarization ratio radar signals and related drop oscillation and propagation effects in rainfall. Radio $S c i$., 19, $81-$ 89.

Ulbrich, C. W., 1981: Effect of size distribution variations on precipitation parameters determined by dual-measurement techniques. Conf. Radar Meteorology, Boston, Amer. Meteor. Soc., 276-281.

- 1983a: Natural variations in the analytical form of the raindrop size distribution. J. Climate Appl. Meteor., 22, 1764-1775.

- , 1983b: An empirical method of accounting for variations in the form of the raindrop size distribution in dual measurement techniques. Conf. Radar Meteorology, Edmonton, Amer. Meteor. Soc., 317-322.

- and D. Atlas, 1984: Assessment of the contribution of differential polarization to improved rainfall measurements. Radio Sci., 19, 49-57. 